\title{
Some recent (and surprising) results on interface and contact line depinning in random media
}

\author{
Jean Vannimenus \\ Laboratoire de Physique Statistique de l'ENS, \\ 24 rue Lhomond, 75005 Paris, France* \\ Version of November 11, 2018
}

\begin{abstract}
I give a brief review of results obtained recently at Ecole Normale on the depinning transition of interfaces and contact lines, using a variety of approaches: non-local Monte Carlo algorithms, dynamical renormalisation group calculations to 2-loop order, and exact solution of an infinite-range model.
\end{abstract}

PACS numbers: 64.60.Lx, 05.40.+j, 05.70.Ln

Keywords: nonequilibrium transition; depinning; contact line; exact solution; Edwards-Wilkinson model; Leschhorn model.

\section{Introduction}

The motion of interfaces in random media (or more generally of "elastic manifolds"), and in particular the existence of a depinning threshold analogous to a critical point, is a now classic problem of statistical mechanics which appears under various forms in many areas of condensed matter physics [1]. In spite of much progress during the last 15 years [2] many interesting questions remain open and I take this opportunity to present some results recently obtained by several groups at Ecole Normale. A personal motivation to talk about this topic here is that among many domains of common interest with Gene Stanley this is one of the few where I can claim priority, having studied contact lines on disordered substrates with Y. Pomeau [3] before Gene's own work on interface depinning [4] - see [5] for relevant references up to 1995.

\footnotetext{
*Laboratoire associé au CNRS et aux Universités Paris VI et Paris VII.
} 
The incentive to look at that problem again came initially from experimental results obtained in our laboratory by E. Rolley and his group on the helium-cesium contact line [6, 0]. A main goal was to improve the determination of the depinning threshold in order to obtain more precise values for the roughness and velocity exponents, in the hope to reconcile numerical simulations with the experimental results and with new theoretical predictions due to LeDoussal and coworkers [8]. As will become apparent from this brief review the results brought some surprises and call for further work.

\section{Algorithms and simulations}

A detailed description of the motion of a fluid-solid contact line is quite intricate if one tries to take into account in a realistic way both the hydrodynamic aspects [9] and the substrate inhomogeneity. For this problem and for interface depinning in general most theoretical studies therefore focus on the effects of disorder close to the threshold, when the mean velocity is sufficiently small for inertial effects to be negligible, at least as a first approximation. It is also customary to disregard the possibility for the interface to develop overhangs in order to pass round unfavorable regions. With these approximations the equation of motion at zero temperature may be written

$$
\partial u / \partial t=K[u]+F_{e x t}+\eta(u),
$$

where $u(\vec{r}, t)$ is the displacement measured from a flat reference $(d-1)$ dimensional interface. $K[u, \vec{r}]$ is an elastic force that tends to keep the interface flat, its form depends on the system considered and is non-local for the contact line. $F_{\text {ext }}$ is an external driving force and $\eta(u)$ represents the interaction with the disordered medium or substrate, it will be restricted in the following to random, spatially uncorrelated pinning forces distributed according to a given probability density $\rho(\eta)$.

An essential difference with the much studied Edwards-Wilkinson and Kardar-Parisi-Zhang growth models [5] is that here the disorder is quenched, as the random part of the force felt by an immobile interface element remains constant in time. This non-linearity makes the problem quite difficult to tackle analytically. Numerical simulations of (1) with continuous space and time also raise technical difficulties 10 . In particular the interface will always get pinned by rare strong local defects if the maximum distorsion $\left|u_{i}-u_{j}\right|$ allowed in a local move is fixed and if the random force is drawn from a continuous distribution, e.g., a Gaussian as used in field-theoretical calculations. Most simulations have in fact been performed on discretized 
cellular-automata type versions of the problem, where the transverse positions $u_{i}\left(\overrightarrow{r_{i}}, t\right)$ of $N$ interface sites are updated at regular time intervals, the jumps being continuous or discrete. Such simulations are rather straightforward to perform, at least in low dimensions, but even for these simplified versions it is not easy to obtain accurate values of the depinning threshold and of the associated critical exponents [11, 12]. The interface velocity becomes extremely small and fluctuates strongly, so very long simulation times and large system sizes are necessary. Another problem is that it is difficult to identify the equation that results in the continuum limit.

This led Rosso and Krauth 13] to consider non-local Monte Carlo algorithms that bypass the dynamics and give direct access to the stationary configurations of the continuum equation (11), in the pinned phase. When the external force is increased the last configuration to get destabilized is the critical interface, it is unique for a finite sample with perodic boundary conditions. Rosso and Krauth showed how this interface may be efficiently determined using an algorithm in which one looks at each time step for the "front" of minimal length that it is favourable to move by one lattice unit. The roughness exponent $\zeta$ for the interface width $w$ as a function of its lateral extension, $w \sim L^{\zeta}$, may thus be obtained right at the critical point with better accuracy than through the direct dynamical approach.

For short-range elastic interactions with bounded distorsions the result is $\zeta \simeq 0.63$ in $d=2$ [14], in very good agreement with the value obtained from simulations on cellular-automata models [1, 15] which had led to the conjecture

$$
\zeta=\nu_{\perp} / \nu_{\|}=0.633 \pm 0.001
$$

where $\nu_{\perp}$ and $\nu_{\|}$are the transverse and longitudinal exponents for directed percolation. On the other hand, if the local distorsions are unconstrained and the restoring force is linear (i.e., the elastic energy is purely quadratic with respect to these distorsions), giving the so-called "quenched EdwardsWilkinson" model, the exponent is found to be $\zeta_{Q E W}=1.17$, in agreement with other earlier studies 10.

If the restoring force is non-linear, as obtained for instance by adding a quartic term of the form $\left(u_{i}-u_{j}\right)^{4}$ in the elastic energy of the QEW model, one recovers $\zeta \simeq 0.63$. This value appears to be robust and to correspond to a broad class of systems. The surprise is that it is obtained in the absence of an anisotropy term [17], while the "standard" value $\zeta \simeq 1.2$ seems to be specific to the harmonic QEW model. It remains to take into account such higher-order terms in the RG approach and to determine precisely the different universality classes and their extent. 


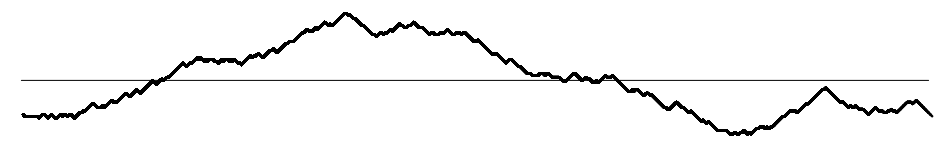

Figure 1: Critical interfaces obtained using the "variant Monte Carlo" algorithm, for short-range interactions with restricted height differences (top) and for long-range interactions (bottom). The size is $N=512$ in both cases (courtesy of A. Rosso).

For the contact line problem the capillary tension acting on the fluid surface leads to an effective long-range interaction between the interface elements [3] and the elastic force has the form

$$
K[u] \propto \int d r^{\prime} \frac{u(r, t)-u\left(r^{\prime}, t\right)}{\left(r-r^{\prime}\right)^{2}} .
$$

The simulations are more time-consuming in this case and a specific algorithm has to be devised, but the critical interface may still be determined accurately [18]. It is found to be much less rough, see figure (1), with

$$
\zeta_{L R}=0.388 \pm 0.002
$$

This result is also surprising, as experiments on well-controlled strongly disordered substrates [7] give $\zeta_{\text {exp }}=0.56 \pm 0.03$, much higher than initially found on weakly disordered but ill-controlled substrates. The calculation of $\zeta$ using dynamical-RG methods is tricky, and the most recent work has shown that contributions at 2-loop order do not vanish [8], contrary to initial expectations. One finds $\zeta^{(2)}=0.47$ at this order, leading to an extrapolated estimate $\zeta_{R G}=0.5 \pm 0.1$, which lies closer to the experimental results but further from the numerical ones than the one-loop value $\zeta^{(1)}=1 / 3[19]$. 


\section{$3 \quad$ An exactly solvable model}

In the case of equilibrium phase transitions the critical exponents of systems with power-law interactions such as (3) are intermediate between those of short-range systems and the mean-field results, which are obtained in the limit of weak uniform infinite-range interactions. The latter are also used as the starting point for field-theory RG treatments and it is natural to study analogous models for moving interfaces [1, 20]. For discretized models the elastic restoring force on site $i$ is simply given in the infinite-range limit by a sum over all sites

$$
K_{i}[u] \propto \frac{1}{N} \sum_{j=1, N}\left(u_{j}-u_{i}\right)=\bar{u}-u_{i},
$$

so the motion of an interface element is just that of a particle in a onedimensional potential, but with an additional coupling to the position $\bar{u}(t)$ of the center of mass, which is to be determined self-consistently.

It is usually assumed in order to obtain analytical solutions that the infinite-range interactions have the effect of averaging out the disorderinduced fluctuations. The interface velocity $v=d \bar{u} / d t$ is then independent of time in the mobile phase, in the limit of an infinite system, and obeys a self-consistent equation. The solution shows that

$$
v \sim\left(F_{\text {ext }}-F_{c}\right)^{\theta}
$$

where $F_{c}$ is the critical force needed to depin the interface. For non-singular distributions of the random pinning forces the critical velocity exponent is found to be [1]

$$
\theta_{M F}=1 .
$$

One would like to have a rigorous proof of this simple and physically reasonable result, but this is not so easy to obtain, as it involves an interchange of limits when time and size go to $\infty$ which may be tricky. Indeed, we have been able to solve exactly a particular model in the infinite-range limit [21] and the exponent found in that case differs from the result (7).

\subsection{The infinite-range Leschhorn model}

This solvable model belongs to a family of cellular-automaton type models due to Leschhorn [11, in which the interface sites may only move forward by a discrete distance at regularly spaced time intervals (synchronous dynamics). We specialize to the case where the positions $u_{i}$ are integer-valued 
and move by one lattice unit at a time:

$$
u_{i}(t+1)=\left\{\begin{array}{lll}
u_{i}(t)+1 & \text { if } & f_{i}(t)>0 \\
u_{i}(t) & \text { if } & f_{i}(t) \leq 0
\end{array}\right.
$$

where $f_{i}$ is the total force on site $i$. In the infinite-range limit it is given by

$$
f_{i}(t)=\bar{u}(t)-u_{i}(t)+F_{e x t}+g \eta_{i}\left(u_{i}\right)
$$

the coupling parameter $g$ controls the strength of the substrate disorder, the distribution $\rho(\eta)$ being normalized and of zero mean.

Even for such a simple system it is not straightforward to determine the threshold very accurately, as for finite $N$ the interface only travels a finite distance $h_{m}$ before it stops. This distance increases rapidly with $N$ in the mobile phase but it fluctuates strongly from sample to sample close to the threshold and the interface velocity is not precisely defined. The stopping distance plays the same role here as the polarization for charge-density waves

22]. For a fixed driving force its average $\left\langle h_{m}\right\rangle$ is expected to follow a scaling law of the form

$$
<h_{m}>\sim N^{\kappa} H\left[N^{y}\left(g-g_{c}\right)\right]
$$

where the scaling function $H(z)$ is finite and regular for $z=0, g_{c}\left(F_{\text {ext }}\right)$ is the critical coupling, and $\kappa$ and $y$ are critical exponents. Expression (10) contains three unknown parameters and it is more practical and accurate to study the Binder ratio

$$
R_{H}=<h_{m}^{2}>/<h_{m}>^{2}
$$

which is expected to converge to a finite value at the critical point when $N \rightarrow \infty$. The results are displayed in figure (21) for a symmetric uniform disorder distribution, $\rho(\eta)=1 / 2$ for $-1<\eta<1$. The critical ratio is $R_{H}^{*} \simeq 1.28$, a little below the value $4 / 3$ which would correspond to a Gaussian distribution for $h_{m}$, and this approach yields an estimate $g_{c}=$ $2.380 \pm 0.005$ for the threshold at $F_{\text {ext }}=0$.

\subsection{Exact solution}

An exact solution of the model may in fact be obtained by noting that deterministic evolution equations can be written down for $P_{k}(x, t)$, the fraction of interface sites at height $k$ which experience a local pinning force $g x$ [21]. 


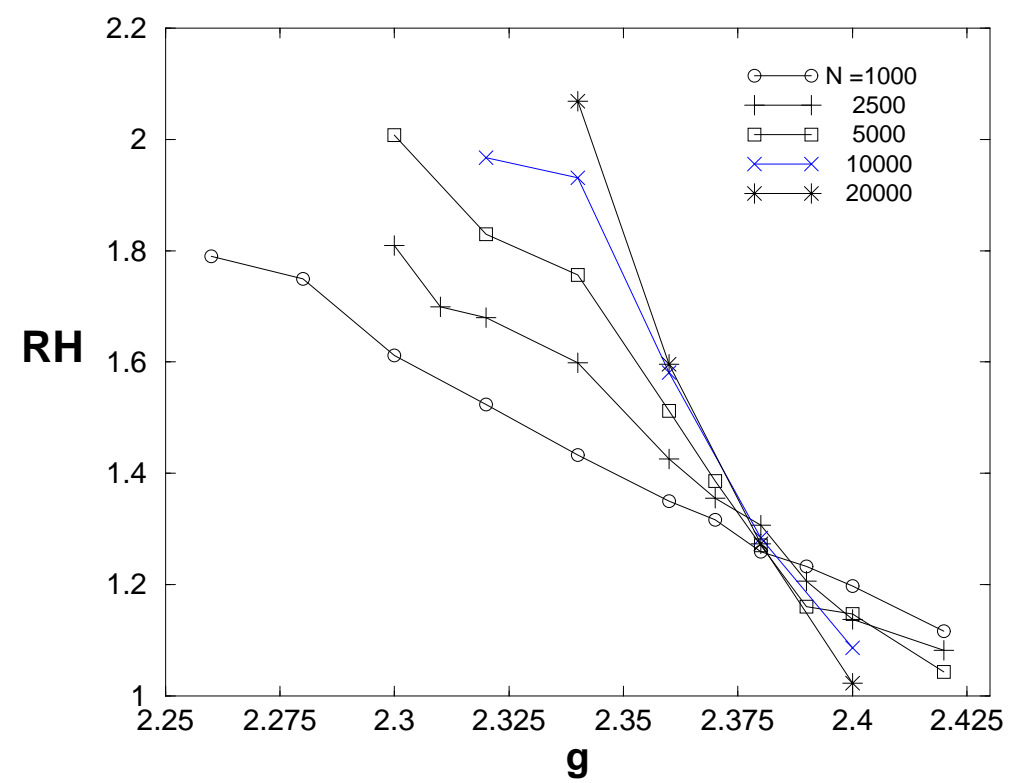

Figure 2: Binder ratio $R_{H}$ for the distribution of stopping distances as a function of the disorder strength $g$ (1000 samples for sizes $N=1000$ to 10000 and 100 samples of size 20000).

The key is to realize that due to the simple dynamics (8) this quantity has a quasi-factorized form :

$$
P_{k}(x, t)=\left\{\begin{array}{lll}
\lambda_{k}(t) \rho(x) & \text { if } & x<\eta_{k}(t), \\
\mu_{k}(t) \rho(x) & \text { if } \quad x>\eta_{k}(t),
\end{array}\right.
$$

where the discontinuity point is related to the mean interface position by $\eta_{k}(t)=(k-\bar{u}(t-1)) / g$. The weights $\lambda_{k}(t)$ and $\mu_{k}(t)$ obey (relatively) simple recursion relations and the end result is a closed system of equations describing the interface dynamics in the thermodynamic limit.

In the mobile phase $\left(g<g_{c}\right)$, this system admits only time-dependent solutions so the threshold may be determined by studying the existence of stationary solutions. For a uniform $\rho(\eta)$ the calculation can be done analytically and the threshold is given by the value of $g$ for which a certain polynomial has a double root. For small driving forces one obtains [21]

$$
g_{c}=2.38006232 \ldots+2.3901 \ldots F_{\text {ext }} .
$$

Note that a threshold exists even for a negative $F_{\text {ext }}$ : Some interface elements will move forward against the external bias, if the local pinning force 
$g \eta_{i}$ acting on them is strong enough, and due to the asymmetry of the dynamics (8) this is not compensated by backward motions of other elements. This "ratchet" effect is of collective origin - an individual particle in a negatively biased random potential would eventually stop -, and it is driven by quenched disorder rather than by random diffusion as in models recently proposed for molecular motors [23].

The threshold may also be obtained more directly through the following argument. Let us assume that in the pinning phase, among all the possible equilibrium positions for an interface element, the one actually reached dynamically is the first lattice site $z^{*}$ for which $f_{i}$ is non-negative [1], i.e.:

$$
\begin{aligned}
f\left(z^{*}\right) & =\bar{z}-z^{*}+g \eta\left(z^{*}\right) & \leq 0, & \\
f(z) & =\bar{z}-z+g \eta(z) & >0, & \text { for all } z<z^{*},
\end{aligned}
$$

where the position $\bar{z}$ of the center of mass can be restricted to $0 \leq \bar{z}<1$ due to statistical translational invariance. The probability density of the stopping point is given by

$$
\mathcal{P}\left(z^{*}\right)=\left[\prod_{z_{i}<z^{*}} R\left(z_{i}\right)\right]\left[1-R\left(z^{*}\right)\right],
$$

where $R(z)$ is the probability that $(z-\bar{z})<g \eta(z)$. For a uniform symmetric distribution $\rho(\eta)$ one has

$$
R(z)= \begin{cases}1 & \text { if } z<\bar{z}-g \\ 0 & \text { if } z>\bar{z}+g \\ \frac{1}{2}\left(1-\frac{z-\bar{z}}{g}\right) & \text { otherwise }\end{cases}
$$

The self-consistency condition on the center of mass, $\bar{z}=\sum z^{*} \mathcal{P}\left(z^{*}\right)$, then yields a polynomial equation in $\bar{z}$ whose coefficients are functions of $g$ and the condition for existence of acceptable solutions can be shown to be identical to the one obtained above for the threshold $g_{c}$.

The exact solution also shows that the interface has a bounded width and the average stopping distance $<h_{m}>$ is finite at $g_{c}$, so

$$
\zeta=\kappa=0
$$

in agreement with standard mean-field predictions. An analysis of the numerical results shown in figure (2) gives a rough estimate $y \sim 0.5$ for the size exponent in (10).

Once the threshold is known exactly it is possible to study the interface dynamics in its vicinity in detail, analytically and numerically. One 
finds that in contrast with the assumption usually made the velocity $v(t)$ is strongly non-uniform: the interface moves through rapid bursts, separating periods of very slow motion. The minimum velocity $v_{\min }$ vanishes linearly but the average velocity $\bar{v}$, measured over a very long time interval, vanishes at the threshold as

$$
\bar{v} \simeq 0.06811 \ldots\left(g_{c}-g\right)^{1 / 2},
$$

which corresponds to a critical exponent

$$
\theta=1 / 2 .
$$

This result is surprising at first sight, as it differs from the standard mean-field prediction, $\theta_{M F}=1$. It means that the existence of an underlying lattice is a relevant perturbation, in the RG sense. This can be understood physically, by noting that mean-field theory predicts the interface to be smooth $(\zeta=0)$, i.e., its width remains comparable to the lattice spacing, so the continuum limit cannot be taken naively. In RG terms the discreteness prevents the effective random potential seen by the interface on large scales from developing a cusp at the origin [8].

It remains to be seen if lattice effects also play a role for systems with long-range interactions such as the contact line. Numerically one finds that the crossover region between a linear and a square-root dependence of the form (19) is rather narrow, so it would be difficult to extract the correct critical behaviour from simulations such as those in section (3.1) - this should be kept in mind when analyzing numerical results in finite dimensions.

I thank B. Derrida and A. Rosso for stimulating discussions, and M. Mézard for suggesting the alternative approach to derive the threshold value.

\section{References}

[1] D.S. Fisher, Phys. Rev B 31, 1396 (1985); D. S. Fisher, "Collective transport in random media: from superconductors to earthquakes", Physics Reports 301, 113-150 (1998).

[2] T. Nattermann, S. Stepanow, L.-H. Tang, H. Leschhorn, J. Physique (France) II 2, 1483 (1992); M. Kardar, "Nonequilibrium dynamics of interfaces and lines", Physics Reports 301, 85-112 (1998); A. Hazareesing, M. Mézard, Phys. Rev. E 60, 1269 (1999); P. Chauve, T. Giamarchi, P. Le Doussal, Phys. Rev B 62, 6241 (2000).

[3] Y. Pomeau, J. Vannimenus, J. Colloid Interface Sci. 104, 477 (1985). 
[4] S.V. Buldyrev et al. Phys. Rev. A 45, R8313 (1992); S.Glotzer et al., Phys. Rev. E 49, 247 (1994).

[5] A.L. Barabási, H.E. Stanley, "Fractal concepts in surface growth" Cambridge U.P. (1995).

[6] E. Rolley, C. Guthmann, R. Gombrowicz, V. Repain, Phys. Rev. Letters 80, 2865 (1998).

[7] A. Prevost, E. Rolley, C. Guthmann, Phys. Rev. B 65, 064517 (2002).

[8] P. Chauve, P. Le Doussal, K. Wiese, Phys. Rev. Letters 86, 1785 (2001).

[9] P. Seppecher, Int. J. Eng. Sci. 34, 977 (1996); L. Pismen, Y. Pomeau, Phys. Rev. E 62, 2480 (2000).

[10] H.J. Jensen, J. Physics A 28, 1861 (1995).

[11] H. Leschhorn, J. Physics A 25, L255 (1992); H. Leschhorn, Physica A 195, 324 (1993); H. Leschhorn, T. Nattermann, S. Stepanow, L.-H. Tang, Ann. Physik 6, 1-34 (1997).

[12] M. Jost, K.D. Usadel, Physica A 239, 486 (1997).

[13] A. Rosso, W. Krauth, Phys. Rev. B 65, 012202 (2002).

[14] A. Rosso, W. Krauth, Phys. Rev. Letters 87, 187002 (2001).

[15] L.-H. Tang, H. Leschhorn, Phys. Rev. A 45, R8309 (1992).

[16] L.A.N. Amaral, A-L. Barabási, H.E. Stanley, Phys. Rev. Letters 73, 62 (1994).

[17] L.-H. Tang, M. Kardar, D. Dhar, Phys. Rev. Letters. 74, 920 (1995).

[18] A. Rosso, W. Krauth, Phys. Rev. E 65, RC025101 (2002).

[19] D. Ertaş, M. Kardar, Phys. Rev. E 49, RC2532 (1994).

[20] J. Koplik, H. Levine, Phys. Rev. B 32, 280 (1985).

[21] J. Vannimenus, B. Derrida, J. Stat. Physics 105, 1 (2001).

[22] A.A. Middleton, D.S. Fisher, Phys. Rev. B 47, 3530 (1993).

[23] F. Jülicher, A. Ajdari, J. Prost, Rev. Modern Phys. 69, 1269 (1997). 